\title{
Publicly Available Pricing Information for Orthopaedic Upper Extremity Procedures in the United States Lacks Transparency and Consistency Between Major Hospitals
}

\author{
M. Lane Moore, B.S., Jordan R. Pollock, B.S., Matthew K. Doan, B.S., Jack M. Haglin, B.S., \\ Kelly L. Scott, M.D., M.P.H., Joshua S. Bingham, M.D., and Karan A. Patel, M.D.
}

\begin{abstract}
Purpose: The purpose of this study was to assess the price variation of various Medicare severity diagnosis-related group codes for orthopaedic upper extremity procedures for the top 20 orthopaedic hospitals using chargemaster price listings from each hospital. Methods: The top 20 orthopaedic hospitals in the United States were determined by querying the U.S. News and World Report's 2020 orthopaedic hospital ranking. This report ranks orthopaedic hospitals according to 4 major domains: outcomes, structure, process/expert opinion, and patient experience. Chargemaster data for the top 20 orthopaedic hospitals was compiled from their websites. Five DRG codes that represented orthopaedic upper extremity procedures were selected, and the pricing information for each was extracted from hospital chargemasters. The median income and cost-of-living index were also compiled for the county that each hospital is located in so that pricing data could be compared to economic measures through regression analysis. Results: Of the top 20 orthopaedic hospitals, 18 had publicly available pricing information in DRG format on their websites. The DRG code with the highest pricing variability was Hand Injury Procedures (DRG 906; range, \$12,832-\$253,633). The procedure with the least pricing variability was Hand or Wrist Procedures (DRG 514; range, \$24,533-\$128,403). Additionally, only the cost of living index was a statistically significant predictor of procedure pricing with a weak correlation. Conclusion: Hospital chargemaster listings are lacking in 2 major areas: true price transparency and standardization/consistency between hospitals. Chargemaster data are often difficult to find, confusing to patients, and inaccurate. Additionally, the price range for a single DRG code can also vary substantially depending on the hospital. It is possible that hospitals located in areas with high costs of living and median incomes would charge higher prices, but these factors were not found to support this hypothesis.
\end{abstract}

I n 2016, Americans had more than 30 million office visits to orthopaedic physicians and 9.5 office visits per 100 persons. ${ }^{1}$ Care for orthopaedic issues accounts for a significant financial burden. In 2018, orthopaedic

From the Mayo Clinic Alix School of Medicine, Mayo Clinic (M.L.M., J.R.P., M.K.D., J.M.H.), Scottsdale, Arizona; and the Department of Orthopedic Surgery, Mayo Clinic (K.L.S., J.S.B., K.A.P.), Phoenix, Arizona, U.S.A.

The authors report the following potential conflicts of interest or sources of funding: K.A.P. reports personal fees from Arthrex, Inc. Full ICMJE author disclosure forms are available for this article online, as supplementary material.

Received October 20, 2021; accepted January 15, 2022.

Address correspondence to M. Lane Moore, B.S., Mayo Clinic Alix School of Medicine, 13400 E Shea Blvd., Scottsdale, AZ 85259, U.S.A. E-mail: moore. michaell@mayo.edu

(C) 2022 THE AUTHORS. Published by Elsevier Inc. on behalf of the Arthroscopy Association of North America. This is an open access article under the CC BY-NC-ND license (http://creativecommons.org/licenses/by-nc-nd/4.0/). 2666-061X/211544

https://doi.org/10.1016/j.asmr.2022.01.005 surgeons collected more than $\$ 3$ billion from Medicare alone, ranking in the top 7 of all specialties. ${ }^{2}$ Additionally, more than 27 million Americans are uninsured, and one third of all Americans will experience financial burden because of medical care at some point in their lives. ${ }^{3,4}$ An unplanned orthopaedic surgery has the potential to be financially devastating to a significant number of Americans. In addition to the high prices charged for healthcare in the United States, little is known about the cost of a medical procedure until the hospital sends the patient the bill after the service has been performed. ${ }^{5}$ Price transparency has been a topic of debate in politics for many years and is proposed as 1 way to alleviate the financial burden on patients. Increased price transparency allows patients receiving medical care the ability to better budget and prepare their finances, apply for financial aid at appropriate times in the treatment course, and make more informed decisions about their medical treatment. 
In 2018, the Centers for Medicare $\&$ Medicaid Services (CMS) issued a ruling to increase price transparency among U.S. hospitals. ${ }^{6}$ This was done in an attempt to lower the continuously rising healthcare costs in the United States by giving healthcare consumers the ability to "shop" for better prices and create a market force that would decrease costs. This initial ruling required all U.S. hospitals to make their healthcare pricing information publicly available by January 2019 in "chargemasters," which are documents listing the standard pricing information for all hospital services. However, this ruling was slow to take effect and many hospitals did not publish this information. In 2019, the Trump administration issued an executive order requiring all hospitals to publish their chargemaster data by January 2021 . $^{7}$

Recently, chargemaster price listings have been criticized for offering inaccurate or misleading information about the true cost of medical services. ${ }^{8,9}$ The prices listed in the chargemasters are prices that a hospital would ideally like to charge a consumer for a given service. Because there is no legislation in place to limit the actual costs of a given procedure or service, these costs may vary substantially and may not be accurate representations of the actual costs. ${ }^{10}$ Accurate pricing information will become increasingly important as demand for upper extremity procedures within orthopaedics continues to rise. For example, as our population ages, the demand for primary total shoulder arthroplasty is projected to increase by nearly $800 \%$ from 2011 to 2030, which is a higher rate of projected growth than total hip or knee procedures. ${ }^{11}$ Given the large volume of orthopaedic procedures being performed in the United States and the large proportion of upper extremity procedures performed within orthopaedics, we sought to investigate this potential variability of chargemaster data. Therefore the purpose of this study was to assess the price variation of various Medicare severity diagnosis-related group (MS-DRG) codes for orthopaedic upper extremity procedures from the top 20 orthopaedic hospitals using chargemaster price listings from each hospital. Our hypothesis is that significant price variation will exist among the top 20 orthopaedic hospitals for the chosen MS-DRG codes; however, regional factors such as cost of living and median income will likely be correlated to the regional pricing differences we expect to observe.

\section{Methods}

Approval from our local Institutional Review Board was deemed exempt for this study because hospital chargemaster data are publicly available and do not contain patient information. In May 2020, the U.S. News and World Report's orthopaedic hospital ranking website was queried to determine the top 20 ranked orthopaedic hospitals in the country. ${ }^{12}$ This report ranks orthopaedic hospitals according to 4 major domains: outcomes, structure, process/expert opinion, and patient experience. ${ }^{13}$ The top 20 hospitals in orthopaedics likely offer similar quality of care and were selected to control for confounding variables. The websites for each of the top 20 ranked orthopaedic hospitals were searched for chargemaster information. Hospitals that had not yet posted this information on their websites or did not include standard pricing for various DRG codes were excluded from the analysis.

Five DRG codes that represented a wide variety of orthopaedic upper extremity procedures were selected and the pricing information for each was extracted from hospital chargemasters (Table 1). The 5 DRG codes selected for this analysis represented procedure groupings that contained orthopaedic procedures dealing with the upper extremity only. Other DRG codes that contained upper extremity procedures under their umbrella were not selected if other, non-upper extremity procedures were also included in their grouping. These DRG codes included the following: “Major Joint/Limb Reattachment Procedures of Upper Extremities" (including total shoulder arthroplasty) (DRG 483), "Major Thumb or Joint Procedures" (DRG 506), "Shoulder, Elbow or Forearm Procedure, Excluding Major Joint Procedures Without Complications/Major Complications" (DRG 512), "Hand or Wrist Procedure, Except Major Thumb or Joint Procedure Without Complications/Major Complications" (DRG 514), and "Hand Procedures for Injuries" (DRG 906). These codes were selected because they were representative of a variety of orthopaedic upper extremity procedures. DRG codes indicating medical complication or major complication were excluded to reduce the risk of introducing confounding variables. For additional context as to what surgical procedures fall under each chosen DRG code, The Centers for Medicare o Medicaid Services Definitions Manual can be referenced. ${ }^{14}$

Chargemaster pricing data were averaged across all hospitals, and basic descriptive statistics were calculated for each DRG code. Additionally, a sub-analysis was performed to determine correlations between chargemaster pricing data and median income or cost of living in the hospitals' respective area. The median income for the county of each respective top ranked orthopaedic hospital was collected from the U.S. Census Bureau's 2018 Median Income report. ${ }^{15}$ The median cost of living for the hospital's county was collected from bestplaces.net cost-of-living database. ${ }^{16}$ DRG pricing information from each hospital was compared to these economic measures through a linear regression analysis.

The accuracy of chargemaster billing information and variation was assessed by comparing the tenth and ninetieth percentile price for each DRG code to the degree of variation that is commonly seen in inpatient 
Table 1. Orthopaedic Upper Extremity DRG Codes and Their Respective Descriptions

\begin{tabular}{ll}
\hline DRG Code & \multicolumn{1}{c}{ Description } \\
\hline 483 & $\begin{array}{c}\text { Major joint/limb reattachment procedure of upper } \\
\text { extremities }\end{array}$ \\
506 & $\begin{array}{l}\text { Major thumb or joint procedures } \\
\text { Shoulder, elbow or forearm procedure, excluding } \\
\text { major joint procedures w/o complications/major } \\
\text { complications }\end{array}$ \\
514 & $\begin{array}{l}\text { Hand or wrist procedure, except major thumb or } \\
\text { joint procedure w/o complications/major } \\
\text { complications }\end{array}$ \\
906 & Hand procedures for injuries \\
\hline DRG, diagnosis-related group.
\end{tabular}

DRG, diagnosis-related group.

commercial claims databases (approximately 50\% to $400 \%) .{ }^{17,18}$ All data collection and analysis were completed using Microsoft Excel (Microsoft Corp, Redmond, WA).

\section{Results}

Of the top 20 orthopaedic hospitals reviewed, 18 $(90 \%)$ had publicly available pricing information for the chosen upper extremity DRG codes in DRG format on their websites. Of the remaining 2 hospitals, one did not have chargemaster data publicly available and the other did not have hospital procedure pricing listed in DRG code format.

The average price for all 5 upper extremity DRG codes included in this analysis was $\$ 78,395$ (range, $\$ 12,832$ $\$ 258,724)$. The DRG code with the greatest pricing variability between hospitals was "Hand Procedures for Injuries (DRG 906)" with an average price of $\$ 102,077$ and a range of $\$ 12,832$ to $\$ 253,633$, representing a difference by a factor of 19.8 . The median price for DRG 906 was $\$ 77,237$ with a standard deviation (SD) of $\$ 79,571$. "Hand and Wrist Procedures, Except Major Thumb or Joint Procedures (DRG 514)" showed the least pricing variability with an average of $\$ 53,889$ and a range of $\$ 24,533$ to $\$ 128,402$, representing a difference by a factor of 5.2. The median price for DRG 514 was $\$ 45,153$ (SD $\$ 29,740$ ). The variability and descriptive statistics for all included DRG codes are represented further in Table 2 and Fig 1.
When all upper extremity DRG codes were averaged and compared to financial measures, median income was not a significant predictor of average procedure pricing $\left(P=.15, R^{2}=0.12\right)$. Additionally, when comparing each of the 5 individual DRG codes to median income, there was no significant association. However, the cost of living index was a statistically significant predictor of procedure pricing with a weak correlation $\left(P<.05, R^{2}=0.23\right)$. However, neither cost of living nor median income were statistically significant predictors of the variation in pricing for individual DRG codes associated with upper extremity procedures (Table 3). Figs 2 and 3 further illustrate the relationship between upper extremity pricing and median income/ cost of living.

The tenth percentile for all included upper extremity DRG codes was \$29,448, whereas the ninetieth percentile was $\$ 151,891$. This represents a difference factor of 5.2, higher than the price difference of $50 \%$ to $400 \%$ that is commonly seen nationally for inpatient procedures. When analyzing individual DRG codes, only DRG 906 showed a difference factor outside the range of $50 \%$ to $400 \%$ (8.6). Table 4 summarizes the tenth percentile, ninetieth percentile, and difference factors for each DRG code.

\section{Discussion}

Chargemaster price variation among the top 20 ranked orthopaedic hospitals had anywhere from a 5fold to a 20-fold difference in prices for upper extremity orthopaedic DRG codes standard deviations and tenth and ninetieth percentile price differences were also substantial reflecting major inconsistencies and variability between the chosen hospitals. Additionally, chargemaster data were difficult to find on hospital websites and often necessitated calling a help line to locate chargemaster information. On average, orthopaedic upper extremity procedure chargemaster prices varied by more than what is commonly seen in the inpatient commercial claims databases. ${ }^{17,18}$ Given the vast level of variation observed within upper extremity chargemaster data, it is unlikely it is achieving its intended purpose of increasing price transparency among patients. Great variation among hospitals, even among those located in the same state or metropolitan

Table 2. Descriptive Statistics for the Five Included Upper Extremity DRG Codes From Top 20 Orthopaedic Hospital Chargemaster Documents

\begin{tabular}{lrccc}
\hline DRG Code & Mean (USD) & Median (USD) & Standard Deviation (USD) \\
\hline 483 & $\$ 101,309.86$ & $\$ 79,736.32$ & $\$ 59,705.42$ & Range (USD) \\
506 & $\$ 57,806.48$ & $\$ 46,645.26$ & $\$ 31,078.75$ & $\$ 210,517.10$ \\
512 & $\$ 70,313.24$ & $\$ 56,834.47$ & $\$ 43,756.53$ & $\$ 111,063.96$ \\
514 & $\$ 53,889.93$ & $\$ 45,153.00$ & $\$ 29,740.49$ & $\$ 148,736.64$ \\
906 & $\$ 102,076.93$ & $\$ 77,237.00$ & $\$ 79,571.02$ & $\$ 240,801.04$ \\
\hline
\end{tabular}

DRG, diagnosis-related group. 


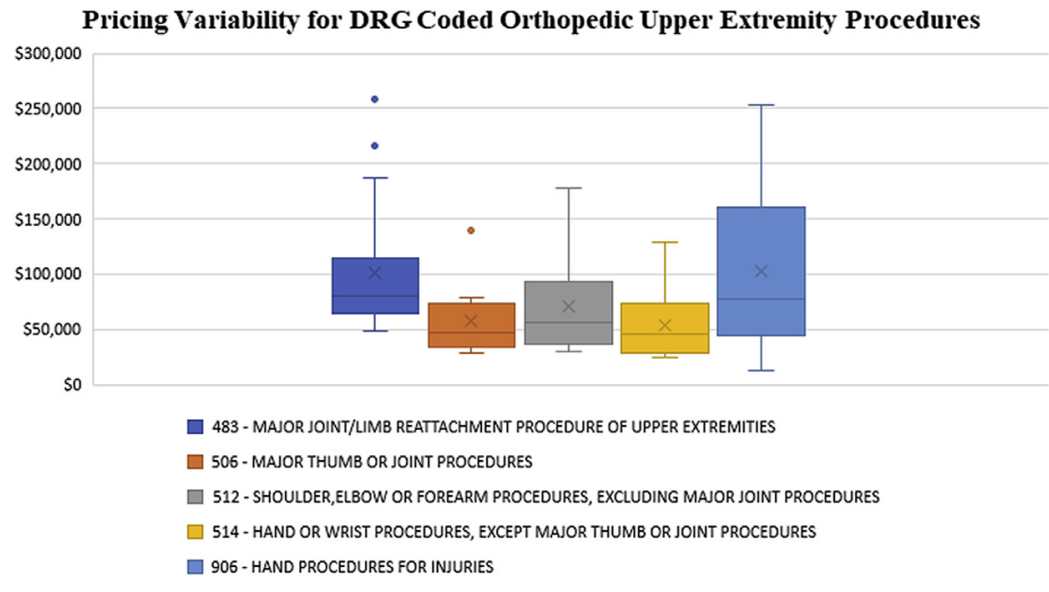

Fig 1. Price variation for the included orthopaedic upper extremity chargemaster diagnosisrelated group (DRG) codes. area, make chargemaster prices an unreliable resource for consumers and likely has little to no effect on driving price competition in healthcare. ${ }^{18} \mathrm{~A}$ recent study by Agarwal et al. ${ }^{19}$ found comparable variation in prostate cancer radiation therapy chargemaster pricing and came to a similar conclusion about the utility of chargemaster pricing.

Access to chargemaster pricing may help to increase the overall price transparency in the U.S. healthcare system, but inconsistent pricing among major hospitals provides little benefit to the average consumer in need of an orthopaedic upper extremity procedure. For example, a patient would need to be very familiar with the healthcare system and the treatment plan to estimate the approximate cost of a procedure. They would need to know the exact procedure being performed,

Table 3. The Relationship Between Cost of Living and Median Income With Orthopaedic Upper Extremity Chargemaster DRG Codes

\begin{tabular}{lll}
\hline & $\mathrm{R}^{2}$ & $P$ value \\
\hline DRG 483 & 0.12 & 0.15 \\
$\quad$ Cost of Living & 0.13 & 0.14 \\
$\quad$ Median Income & & \\
DRG 506 & 0.06 & 0.45 \\
$\quad$ Cost of Living & 0.04 & 0.54 \\
$\quad$ Median Income & & \\
DRG 512 & 0.09 & 0.25 \\
$\quad$ Cost of Living & 0.09 & 0.25 \\
$\quad$ Median Income & & \\
DRG 514 & 0.24 & 0.06 \\
$\quad$ Cost of Living & 0.14 & 0.17 \\
$\quad$ Median Income & & \\
DRG 906 & 0.26 & 0.06 \\
$\quad$ Cost of Living & 0.27 & \\
$\quad$ Median Income & & 0.27 \\
All DRG Codes & 0.23 & \\
$\quad$ Cost of Living & 0.13 & \\
$\quad$ Median Income & & \\
\hline DRG, diagnosis-related group. & & \\
$\quad$ *Indicates significance. &
\end{tabular}

drugs being administered, testing, physician charges, and facility fees. ${ }^{20}$ Even if an educated patient could calculate the cost of medical care through chargemaster data, the prices listed on chargemasters are rarely the price patients pay out of pocket after insurance. Medicare, Medicaid, and private insurers individually negotiate prices with hospitals, and these negotiations often do not reflect charges listed. Although chargemaster pricing can help estimate a procedure's cost between hospitals, the healthcare consumer will almost certainly pay a substantially different sum in the end. Furthermore, modern chargemasters are often difficult to interpret, because procedures tend to be listed in components such as by facility fees, surgeon fees, anesthesia fees, and follow-up care with often unintelligible acronyms that make it difficult for patients to summarize total price estimates. ${ }^{21}$

Although it is unreasonable to expect patients to shop around at different hospitals during medical emergencies, there are medical services that can be planned. For example, in 2016 the Health Care Cost Institute estimated that $43 \%$ of the $\$ 524$ billion spent on healthcare in the United States by individuals with employeesponsored insurance was spent on shoppable services. ${ }^{22}$ For chargemasters to be more useful to patients, we suggest publishing predicted out-of-pocket charges for patients stratified by insurance company and plan. Otherwise, listing general charges of medical services are of little to no benefit to patients. This will be increasingly important as our aging population continues to use these elective services, such as total shoulder arthroplasty. With increased transparency and price understanding, it is thought that market dynamics for these elective procedures will encourage competition, thus resulting in prices becoming more uniform and reasonable.

The purpose behind the use of DRG codes is to ensure that Medicare reimbursement values are an accurate representation of a patient's medical complexity, the 
Fig 2. The association between median income and average orthopaedic upper extremity chargemaster price.
Association of Median Income to Chargemaster Price

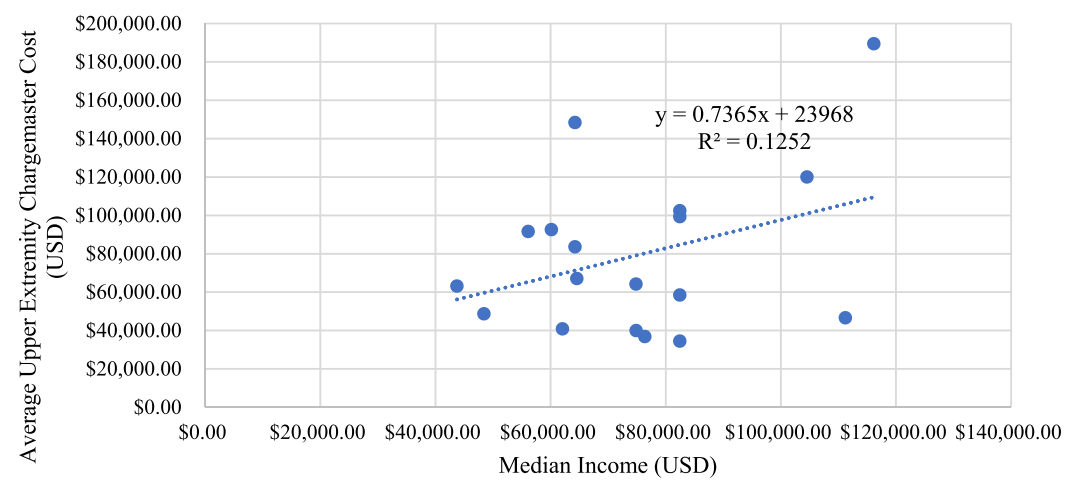

hospital's mix of cases, the type of patients that a particular hospital treats, and the amount of resources that the hospital needs to treat its patients. ${ }^{23}$ Reimbursement values for a particular DRG code reflect the diagnosis and the hospital's base payment rate. The base payment rate for each hospital accepting Medicare in the country is slightly different and can be broken down into a labor component and a non-labor component. ${ }^{23}$ Both of these components are designed to reflect geographical variation in wage-index (labor portion) and cost-of-living (non-labor portion). ${ }^{23}$ Therefore it is interesting to note that regional variations in DRG code reimbursement should vary directly with economic measures like wage-index and cost-ofliving. However, when chargemaster pricing is examined, this relationship is only weakly supported. Regardless of the price listed on a chargemaster, hospitals receive similar reimbursement from Medicare for a particular DRG code.

To achieve this goal of relative price uniformity and transparency, several strategies may be used to expand upon the current CMS-mandated release of chargemaster data. Potential examples of effective pricing transparency can be found in ambulatory surgical centers offering direct-payment (cash) prices online.
These centers are influenced by competition and open market dynamics and they voluntarily share this information in an organized manner to best inform their patient consumers. Consequently, these surgery centers serve as important models for improvement in the design of delivering mandated price disclosures in several ways. First, pricing is advertised via a single, bundled payment covering all aspects of a specific procedure. Second, price estimates are offered both in a cash pay price (exactly what the patient would pay without insurance) and an estimate of what insurance would cover based on historic and geographic payment data in that region. This combination of information gives the consumer an accurate idea of what they will owe and also allows comparison of cost between centers. Theoretically, this pricing transparency could drive prices down and encourage price uniformity. ${ }^{21}$

The CMS is working to improve the current chargemaster mandate to potentially associate such suggestions. In a recently released statement, the CMS acknowledged that the current chargemaster mandate does not reflect accurate information for insured patients but is "an important first step to increase price transparency." Although the CMS plans to mandate more accurate and organized pricing information in the
Association of Cost of Living Index to Chargemaster Price
Fig 3. The association between the cost of living index and average orthopaedic upper extremity chargemaster price. 
Table 4. Tenth and Ninetieth Percentile Values for Each Orthopaedic Upper Extremity Chargemaster DRG Code and the Difference Factor Between the Tenth and Ninetieth Percentile Values

\begin{tabular}{lcrc}
\hline DRG Code & 10th Percentile & 90th Percentile & Difference Factor \\
\hline 483 & $\$ 57,181.03$ & $\$ 196,367.68$ & 3.4 \\
506 & $\$ 32,361.24$ & $\$ 77,815.30$ & 2.4 \\
512 & $\$ 32,892.68$ & $\$ 125,410.63$ & 3.8 \\
514 & $\$ 27,848.75$ & $\$ 86,909.40$ & 3.1 \\
906 & $\$ 26,956.08$ & $\$ 231,291.48$ & 8.6 \\
All Codes & $\$ 29,448.60$ & $\$ 151,891.20$ & 5.2 \\
\hline
\end{tabular}

DRG, diagnosis-related group.

future, they encourage hospitals to exceed the mandate's requirements and adopt strategies to help patients understand what their care will cost. ${ }^{24}$

Increased price transparency will also allow increased ability to recognize and address price variation across healthcare systems and geographic locations. It might be fair to assume that chargemaster price differences exist between DRG codes performed at a small, local hospital versus those at a top ranked academic medical center. However, it is difficult to explain the substantial variation seen among the top ranked hospitals included in this analysis. These hospitals likely provide similarly advanced, high-level treatment. In 2013, the Pacific region (Washington, Oregon, California, Alaska, Hawaii) and Middle Atlantic region (New York, New Jersey, Pennsylvania) were found to have significantly higher inpatient charges per stay at $37 \%$ and $16 \%$ higher than the national average, respectively. ${ }^{25}$ In our analysis, 11 of the 20 top ranked orthopaedic hospitals came from these two regions (6 from Middle Atlantic and 5 from Pacific). Additionally, these regions and the major metropolitan areas within them, are among the most expensive places to live in the United States. ${ }^{26}$ However, when the orthopaedic upper extremity chargemaster pricing data was compared to median income and cost of living index, neither cost of living nor median income were strong predictors of these pricing variations. The unexplained variability in chargemaster pricing warrants further investigation.

\section{Limitations}

This study has some limitations. As mentioned previously, many factors and expenses make up an orthopaedic upper extremity procedure. Although the procedure itself is often the focus and the costliest component, we were not able to account for the price of medications, physician charges, imaging, and diagnostic testing. These other charges would inevitably lead to a higher total cost than what is analyzed in this study. We were also limited in our ability to precisely measure chargemaster price transparency because that would entail obtaining the true prices that insurance companies and patients pay for a given procedure. This information is often difficult to find or protected by hospitals or insurance companies.

\section{Conclusion}

Hospital chargemaster listings are lacking in 2 major areas: true price transparency and standardization/ consistency between hospitals. Chargemaster data are often difficult to find, confusing to patients, and inaccurate. Additionally, the price range for a single DRG code can also vary substantially depending on the hospital. It is possible that hospitals located in areas with high costs of living and median incomes would charge higher prices, but these factors were not found to support this hypothesis.

\section{References}

1. NAMCS/NHAMCS - Web Tables. https://www.cdc.gov/ nchs/ahcd/web_tables.htm. Accessed July 2, 2020.

2. Medicare physician expenditures by top specialties 2018 . Statista. https://WwW.statista.com/statistics/298467/ physicians-medicare-collections/. Accessed August 9, 2020.

3. Bureau UC. Health Insurance Coverage in the United States: 2018. The United States Census Bureau. https:// WwW.census.gov/library/publications/2019/demo/p60-267. html. Accessed July 2, 2020.

4. Products - NHIS Early Release Program - Homepage. https://www.cdc.gov/nchs/nhis/releases.htm. Accessed July 2, 2020.

5. Sanger-Katz M. In the U.S., an Angioplasty Costs $\$ 32,000$. Elsewhere? Maybe \$6,400. The New York Times, 27 December 2019. https://www.nytimes.com/2019/12/27/ upshot/expensive-health-care-world-comparison.html. Accessed August 23, 2020.

6. CMS finalizes changes to empower patients and reduce administrative burden | CMS. https://WwW.cms.gov/ newsroom/press-releases/cms-finalizes-changes-empowerpatients-and-reduce-administrative-burden. Accessed July 2, 2020.

7. Executive Order on Improving Price and Quality Transparency in American Healthcare to Put Patients First. The White House. https://www.whitehouse.gov/presidentialactions/executive-order-improving-price-quality-transpa rency-american-healthcare-put-patients-first/. Accessed July 2, 2020.

8. Batty M, Ippolito B. Mystery of the chargemaster: Examining the role of hospital list prices in what patients actually pay. Health Aff (Millwood) 2017;36:689-696.

9. Furlow B. Skepticism about new US Government hospital pricing transparency rule. Lancet Oncol 2019;20:188.

10. Wallack AR, Fournier D, Riley T, et al. Can we please stop fixating on hospital chargemasters? The National Academy for State Health Policy. https://www.nashp.org/can-weplease-stop-fixating-on-hospital-chargemasters/. Accessed July 2, 2020.

11. Padegimas EM, Maltenfort M, Lazarus MD, et al. Future patient demand for shoulder arthroplasty by younger 
patients: National projections. Clin Orthop 2015;473: 1860-1867.

12. Best Hospitals for Orthopedics | Rankings \& Ratings | US News Best Hospitals. https://health.usnews.com/besthospitals/rankings/orthopedics. Accessed July 5, 2020.

13. FAQ. How and why we rank and rate hospitals. US News $\theta$ World Report. https://health.usnews.com/health-care/ best-hospitals/articles/faq-how-and-why-we-rank-andrate-hospitals. Accessed July 5, 2020.

14. ICD-10-CM/PCS MS-DRG v37.0 Definitions Manual. https://www.cms.gov/icd $10 \mathrm{~m} /$ version37-fullcode-cms/ fullcode_cms/P0001.html. Accessed January 5, 2022.

15. Explore Census Data. https://data.census.gov/cedsci/. Accessed July 5, 2020.

16. 2020 Cost of Living Calculator. https://www.bestplaces. net, https://www.bestplaces.net/cost-of-living/. Accessed July 5, 2020.

17. Cooper Z, Craig SV, Gaynor M, et al. The price ain't right? Hospital prices and health spending on the privately insured. Q J Econ 2019;134(1):51-107.

18. Koller CF, Khullar D. The commercial differential for hospital prices: Responses from states and employers. JAMA 2019;322:723-724.

19. Agarwal A, Dayal A, Kircher SM, et al. Analysis of price transparency via National Cancer Institute-Designated cancer centers' chargemasters for prostate cancer radiation therapy. JAMA Oncol 2020;6:409-412.

20. Hospital Pricing Varies Greatly on Published Chargemasters | Dark Daily. https://wwww.darkdaily.com/ california-healthline-report-finds-hospital-chargemasterprices-fluctuate-dramatically-even-among-hospitals-locatednear-each-other/. Accessed July 7, 2020.

21. Bai G, Patel P, Makary M, et al. Providing Useful Hospital Pricing Information To Patients: Lessons From Voluntary Price Disclosure | Health Affairs. https://www. healthaffairs.org/do/10.1377/hblog20190416.853636/full/. Accessed August 9, 2020.

22. Health Care Cost Institute. Spending on Shoppable Services in Health Care. https://www.healthcostinstitute.org/ images/easyblog_articles/ 1 10/Shoppable-Services-IB-3.2. 16_0.pdf(2016). Accessed August 9, 2020.

23. Health Insurance: How Does a DRG Determine What a Hospital Gets Paid? Verywell Health. https://Www. verywellhealth.com/how-does-a-drg-determine-how-mucha-hospital-gets-paid-1738874. Accessed August 23, 2020.

24. HealthPayerIntelligence. Verma: Price Transparency Rule a "First Step" for Consumerism. HealthPayerIntelligence. https://healthpayerintelligence.com/news/verma-pricetransparency-rule-a-first-step-for-consumerism.

Accessed August 9, 2020.

25. Geographic Variation in Hospital Inpatient List Prices in the United States, 2013 \#209. https://www.hcup-us.ahrq. gov/reports/statbriefs/sb209-Geographic-Variation-Hospital-Inpatient-Prices.jsp. Accessed July 7, 2020.

26. Goetz L. Top 10 Most Expensive Cities in the U.S. Investopedia. https://www.investopedia.com/articles/personal-finance/ 080916/top-10-most-expensive-cities-us.asp. Accessed July 7, 2020. 